Ulaş Serkan Topaloğlu*, Hatice Kayış Topaloğlu, Melih Kızıltepe, Mesut Kılıç, Sami Bahçebaşı, Sibel Ata, Şeyma Yıldız and Yasin Şimşek

\title{
Fear of hypoglycemia in adults with diabetes mellitus switching to treatment with IDegAsp co-formulation to examine real-world setting: an observational study (The HATICE study)
}

https://doi.org/10.1515/dmdi-2020-0166

Received October 9, 2020; accepted December 6, 2020;

published online December 24, 2020

\section{Abstract}

Objectives: To evaluate the clinical results of insulin degludec/aspart (IDEgAsp) therapy and its effect on the fear of hypoglycemia.

Methods: A prospective observational study has been conducted through surveys of 36 patients using insulin because of type 2 diabetes mellitus who initiated treatment with IDegAsp switching from other insulins. Patients, 18-75 years old, were recruited to the study, consecutively. Participants' age, gender, height, weight, body mass index (BMI), daily insulin dose, glycated hemoglobin $\left(\mathrm{HbA}_{1 \mathrm{c}}\right)$, hypoglycemia rate, hypoglycemia fear survey (HFS) were recorded at the beginning of the study. By the end of 12th month, data was re-measured and compared with each other.

Results: $\mathrm{HbA}_{1 \mathrm{c}}$ was declined by mean of $-1.59 \%$ (95\% CI -1.06 to $-2.12, \mathrm{p}<0.001)$. There was also a significant decrease in mean, daily insulin dose, weight and BMI values of patients via IDegAsp. While there was an increase in the amount of dipeptidyl peptidase 4-inhibitors (DPP4-i) and sodium-glucose co-transporter 2-inhibitors (SGLT2-i),

\footnotetext{
*Corresponding author: Ulaş Serkan Topaloğlu, MD, Department of Internal Medicine, Kayseri City Hospital, Molu Street, 38080 Kocasinan/Kayseri, Kayseri, Turkey, Phone: +903523157700 (ext 10248); +90 55555790 16, E-mail: ustop38@gmail.com Hatice Kayış Topaloğlu, Department of Family Medicine, Erciyes University Medical Faculty, Kayseri, Turkey. https://orcid.org/00000002-8836-7012

Melih Kızıltepe, Mesut Kılıç, Sami Bahçebaşı, Sibel Ata and Şeyma Yıldız, Department of Internal Medicine, Kayseri City Hospital, Kayseri, Turkey. https://orcid.org/0000-0001-6100-2024 (M. Kızıltepe). https://orcid.org/0000-0003-0397-4276 (M. Kılıç). https://orcid.org/ 0000-0003-0909-7024 (S. Bahçebaşı). https://orcid.org/0000-00032935-5684 (S. Ata). https://orcid.org/0000-0001-7560-8203 (Ş. Yıldız) Yasin Şimşek, Department of Endocrinology, Kayseri City Hospital, Kayseri, Turkey. https://orcid.org/0000-0003-1654-6422
}

there was a decrease in daily injection frequency. There was also a significant decrease in the median values of monthly hypoglycemia rate (from 2.0 to $1.0, p<0.001$ ) and the entire HFS scores (HFS-T: from 1.09 to $0.73, \mathrm{p}<0.001$; HFS-B: from 0.83 to $0.60, \mathrm{p}<0.001$; HFS-W: from 1.33 to $0.88, \mathrm{p}<0.001)$. There was a strong positive correlation between $\triangle$ HFS-B and daily injection frequency (Rho: 0.398 ; P: 0.016).

Conclusions: IDegAsp co-formulation, combined with DPP4-i and/or SGLT2-i, can provide usefulness in terms of rates of hypoglycemia, reduced $\mathrm{HbA}_{1 \mathrm{c}}$, less injection administration, and decreased the fear of hypoglycemia in diabetics.

Keywords: glycated hemoglobin $\left(\mathrm{HbA}_{1 \mathrm{c}}\right)$; hypoglycemia fear; insulin degludec/aspart (IDegAsp).

\section{Introduction}

Diabetes mellitus is a progressive complex disease, and achieving normoglycemia is the first goal in patients with diabetes. On the way to achieving the target, it is necessary to increase the quality of life of the patients, reduce the complications and consider the comfort of the patient while performing this [1,2]. There are many oral and subcutaneous medications that provide these opportunities in the struggle against diabetes. As novel drugs and new regimens are added to diabetes treatment day by day, we should be able to make the right choice meeting the patient's standards [3].

Premixed therapies currently available are commonly administered in Asia based on the fact that the basalbolus regimen requires multiple daily administration [4]. Nevertheless, the protaminated fraction of pre-mixed insulin interferes with the soluble fraction and thus, resulting in "shoulder effect" prolonging the effect of the soluble fraction. This effect may lead to increased hypoglycemia, extra food consumption and weight gain [5]. Occasionally, this problem can be prevented with basal (and plus) insulin-supported oral therapy instead of 
pre-mixed one. Also, the basal-plus strategy offers an effective alternative to a full basal-bolus regimen and may help to overcome barriers associated with multiple injections and expected complexity of the insulin administration [6].

Administered as a soluble co-formulation of $70 \%$ insulin degludec (basal coverage) and 30\% insulin aspart (postprandial coverage), insulin degludec/aspart (IDegAsp) eliminates the anxiety caused by the shoulder effect and offers basal-plus treatment in a single pen $[3,7]$. There is a scarcity of studies and limited data in the literature on the observational outcomes of IDegAsp treatment. In this study, we aimed to reveal the observational results of IDegAsp treatment supported oral therapy over the 12-month-period. In the meanwhile, we aimed to evaluate the effects on the fear of hypoglycemia with the hypoglycemia fear survey (HFS).

\section{Materials and methods}

\section{Study design}

The clinical trial protocol was approved by the Ethics Committee of Erciyes University and complied with the Declaration of Helsinki and Good Clinical Practice. The patients' written informed consents to publish the clinical information and materials was obtained. This was a prospective observational study of data obtained by specialists' surveys of 36 patients. The study has been conducted in 2019 between January and December. The inclusion criteria for physicians were having the specialist certification in internal medicine, more than two years in practice, at least $50 \%$ of their time spent managing patients in a clinic, treating a minimum of 10 patients with diabetes on a weekly basis, and having treated a minimum of three patients with IDegAsp in the past 6 months.

Patients, aged between 18 and 75 years, admitted to a tertiary hospital's internal medicine outpatient clinic for routine diabetes mellitus control were recruited to the study, consecutively. Patients using insulin because of type 2 diabetes mellitus who started treatment with IDegAsp switching from other insulins were included in the study. The participants who had a prior diagnosis active malignancy, alcohol intake, liver cirrhosis, psychic disease (such as bipolar disorders and schizophrenia), adrenal or pituitary disease (such as pheochromocytoma, Cushing and Addison) and administering corticosteroids were excluded. Moreover, thyroid function tests of the patients were examined, and those who were not euthyroid were excluded from the study. Accordingly, the confounding factors affecting the working conditions by frequent variation in blood glucose level were prevented. Participants' age, gender, height, weight, body mass index (BMI), duration of diabetes, duration of insulin treatment, daily insulin dose, chronic kidney disease epidemiology collaboration (CKD-EPI) for the determination of the glomerular filtration rate, glycated hemoglobin $\left(\mathrm{HbA}_{1 \mathrm{c}}\right)$, hypoglycemia rate, the scale of HFS in the form of a survey, status of oral antidiabetics and insulin administration have been recorded at the beginning of the study. During the insulin switch, addition of dipeptidyl peptidase 4-inhibitors (DPP4-i) or sodium-glucose co-transporter 2-inhibitors (SGLT2-i) to patients deemed appropriate while continuing metformin therapy. Patients using other oral antidiabetics and glucagon-like peptide-1 receptor agonists were excluded from the study. The BMI of the patients was in the range of $20-35 \mathrm{~kg} / \mathrm{m}^{2}$. The patients were informed on diet in accordance with IDegAsp while switching. Twelve months after switching the treatment with IDegAsp patients were only contacted by phone for diabetes control. No extra attention was given to the participants, except for routine diabetes follow-up for 12 months. By the end of 12th month, data was remeasured and compared with each other.

\section{Measurement of laboratory parameters}

A fasting venous blood sample was collected after an overnight fasting for at least 8-h for biochemical examinations and samples have been processed at the hospital laboratory on the same day. Biochemical tests were estimated by using a Roche Cobas 8000 immunoassay analyzer (Roche Diagnostics, USA). $\mathrm{HbA}_{1 \mathrm{c}}$ were estimated by using an Adams A1c HA-8180V automatic analyzer (Arkray Diagnostics, USA). The entire assays were performed with specific kits and calibrators supplied by the relevant manufacturers.

\section{Definitions of hypoglycemia}

The assessment of hypoglycemia included the following definition: an episode requiring assistance of another person to actively administer carbohydrate, glucagon, or/and take other actions; plasma glucose concentrations may not be available; however, neurological improvement following reversal of the blood glucose back to normal values is to be considered as a sufficient proof of a serious episode, or symptomatic plasma glucose confirmed $(<56 \mathrm{mg} / \mathrm{dL})$ episodes [3]. Pre-switch/post-switch data on hypoglycemia was based on the specialists' responses to the following question: "How many hypoglycemia did this patient declare to be experienced in the past month in preswitch and post-switch?” Both responses were counted and compared with each other.

\section{Fear of hypoglycemia}

Fear of hypoglycemia can be scaled using the HFS, published in 1987, which quantifies HFS-Total (HFS-T), HFS-behavior (HFS-B) and HFS-Worry (HFS-W) in patients with diabetes [8]. In HFS-B, consisting of 15 questions, individuals with diabetes are asked questions on what they have done in their daily lives to prevent their blood glucose levels from falling in the past 3 months. In HFS-W, consisting of 18 items, individuals are asked how often they are concerned about the items given depending on the decrease in blood glucose level in the last 3 months. HFS-T is half of the total scores of HFS-B and HFS-W. 0 points: never; 1 point: rarely; 2 points: sometimes; 3 points frequently; 4 points: always in responses to questions. The higher score obtained, the higher fear of hypoglycemia is. The HFS has been often used in clinical trials, and has been translated into more than 50 languages, revealing its validity and reliability [9]. HFS scores was calculated just before switching and 12-months after IDegAsp initiated. 
Table 1: Change in $\mathrm{HbA}_{1 \mathrm{c}}$, weight, BMI, daily insulin dose and CKD-EPI $(\mathrm{n}=36)$.

\begin{tabular}{|c|c|c|c|c|}
\hline & Baseline (mean \pm SD) & 12 months (mean \pm SD) & $\begin{array}{r}\text { Change from baseline } \\
\text { (\%95 Cl) }\end{array}$ & p-Value \\
\hline $\mathrm{HbA}_{1 \mathrm{c}}, \%$ & $9.93 \pm 1.64$ & $8.34 \pm 1.30$ & $-1.59(-1.06,-2.12)$ & $<0.001$ \\
\hline Weight, kg & $85.08 \pm 13.60$ & $82.56 \pm 12.29$ & $-2.53(-0.73,-4.32)$ & 0.007 \\
\hline $\mathrm{BMI}, \mathrm{kg} / \mathrm{m}^{2}$ & $32.54 \pm 5.69$ & $31.65 \pm 5.76$ & $-0,89(-0.24,-1.54)$ & 0.009 \\
\hline Insulin dose/daily, units & $74.94 \pm 34.69$ & $62.22 \pm 18.51$ & $-12.72(-0.87,-24.56)$ & 0.036 \\
\hline CKD-EPI, ml/dk/1.73 m² & $77.06 \pm 22.51$ & $80.89 \pm 22.58$ & $+3.83(+8.75,-1.08)$ & 0.122 \\
\hline
\end{tabular}

$\mathrm{HbA}_{1 \mathrm{c}}$, glycated hemoglobin; BMI, body mass index; CKD-EPI, chronic kidney disease epidemiology collaboration; SD, standard deviation; $\mathrm{Cl}$, confidence interval. $p<0.05$ is considered as statistically significant (bold).

\begin{abstract}
Statistical analysis: Statistical analyses have been performed with the SPSS software version 22.0 (IBM Corp., Armonk, NY, USA). Parametric variables were presented as means and standard deviations, nonparametric variables were presented as medians and interquartile ranges. Shapiro-Wilks test and histograms analyses were used to determine whether continuous variables were normally distributed. Whereas two dependent groups of parametric variables were compared using paired t-test, non-parametric ones were compared using Wilcoxon test. Number of cases and percentages were used for categorical variables. Categorical data has been analyzed by Chi-square. Relationship between non-parametric variables has been analyzed by Spearman correlation tests; a p-value $<0.05$ was considered as a statistically significant difference.
\end{abstract}

\section{Results}

The mean age of the patients was $60.9 \pm 10.6$ years, and also the mean duration of diabetes was $15.1 \pm 7.5$ years. The median value of the time patients used insulin was 8 years. At the beginning of the study, 29 of the 36 patients had been treated with a basal-bolus insulin regimes, while six patients have administered via pre-mixed insulin and only 1 patient has used basal insulin as stand-alone. After the switch, the entire patients were treated with IDegAsp before both breakfast and dinner, while eight patients were also received IAsp treatment before lunch.

At the beginning of the study, six of the 36 patients $(16.7 \%)$ did not use any oral antidiabetic, while 18 of them (50\%) used single and 12 patients (33.3\%) were using double oral antidiabetic agents. At the end of the study, five patients $(13.9 \%)$ did not take any oral antidiabetic drugs due to intolerance and chronic renal failure, while 4 patients $(11.1 \%)$ were using single, 22 patients $(61.1 \%)$ were using double, and five patients (13.9\%) were using triple oral antidiabetic.

There was a significant decrease in mean $\mathrm{HbA}_{1 \mathrm{c}}$, daily insulin dose, weight and BMI values of patients after 12 months IDegAsp treatment. Although there was an increase in the mean CKD-EPI value at the end of the study, it was not statistically significant (Table 1).
Table 2: Change in amount of oral antidiabetics, daily injection frequency, hypoglycemia rate and HFS scores $(n=36)$.

\begin{tabular}{lrrr}
\hline & $\begin{array}{r}\text { Baseline } \\
\text { median (IQR) }\end{array}$ & $\begin{array}{rlr}\text { 12 months } \\
\text { median (IQR) }\end{array}$ & p-Value \\
\hline Amount of oral antidiabetics & $1.0(1.0)$ & $2.0(1.0)$ & $<0.001$ \\
Daily injection frequency & $4.0(0.0)$ & $2(0.0)$ & $<0.001$ \\
Hypoglycemia rate/monthly & $2.0(8.0)$ & $1.0(1.0)$ & $<0.001$ \\
HFS-total & $1.09(0.67)$ & $0.73(0.66)$ & $<0.001$ \\
HFS-behavior & $0.83(1.18)$ & $0.60(0.98)$ & $<0.001$ \\
HFS-worry & $1.33(0.69)$ & $0.88(0.38)$ & $<0.001$ \\
\hline
\end{tabular}

HFS: Hypoglycemia Fear Survey; IQR: Interquartile range. $p<0.05$ is considered as statistically significant (bold).

While there was an increase in the amount of oral antidiabetics, there was a decrease in daily injection frequency. There was also a significant decrease in the median values of monthly hypoglycemia rate and all of the HFS scores (Table 2).

There was a strong positive correlation between $\triangle$ HFS-B and daily injection frequency. $\triangle$ HFS scores and its associations were summarized in Table 3.

\section{Discussion}

In this study, clinical aspects obtained 12 months after treatment with IDegAsp switching from other insulins and evaluation of the fear of hypoglycemia of patients with diabetes has been performed among Turkish people in Kayseri City Hospital, which is located in central Anatolia (also called Cappadocia region in which diabetes was first described and named by Arateus 2000 years ago). Kayseri City Hospital, serving to approximately 4 million people, is one of the largest hospitals in Turkey. Each month, more than 6000 patients with diabetes mellitus are treated in our center. This hospital in which our study was conducted, is a very substantial center for the prevention of diabetes where the entire diabetic complications can be treated. Because of 
Table 3: Correlation analysis of $\triangle \mathrm{HFS}$ scores.

\begin{tabular}{|c|c|c|c|c|c|c|}
\hline & \multicolumn{2}{|c|}{$\Delta$ HFS-total } & \multicolumn{2}{|c|}{$\Delta$ HFS-behavior } & \multicolumn{2}{|c|}{$\Delta$ HFS-worry } \\
\hline & Rho & p-Value & Rho & p-Value & Rho & p-Value \\
\hline Age & -0.224 & 0.188 & -0.327 & 0.051 & 0.088 & 0.611 \\
\hline Duration of diabetes & 0.162 & 0.345 & -0.026 & 0.882 & 0.226 & 0.185 \\
\hline Duration of insulin treatment & 0.246 & 0.147 & -0.046 & 0.791 & 0.250 & 0.142 \\
\hline$\Delta \mathrm{BMI}$ & 0.115 & 0.504 & 0.260 & 0.126 & -0.154 & 0.371 \\
\hline$\Delta$ Weight & 0.114 & 0.508 & 0.252 & 0.137 & -0.143 & 0.407 \\
\hline$\triangle C K D-E P I$ & -0.002 & 0.989 & -0.132 & 0.443 & 0.105 & 0.543 \\
\hline$\Delta \mathrm{HbA}_{1 c}$ & -0.008 & 0.962 & -0.256 & 0.133 & 0.101 & 0.560 \\
\hline$\Delta$ Insulin dose & -0.149 & 0.385 & 0.104 & 0.548 & -0.159 & 0.356 \\
\hline$\Delta$ Amount of oral antidiabetics & -0.150 & 0.381 & -0.181 & 0.290 & 0.000 & 0.999 \\
\hline$\Delta$ Daily injection frequency & 0.253 & 0.137 & 0.398 & 0.016 & 0.045 & 0.795 \\
\hline$\Delta$ Hypoglycemia rate & 0.004 & 0.980 & 0.142 & 0.410 & -0.180 & 0.295 \\
\hline
\end{tabular}

HFS: Hypoglycemia Fear Survey; BMI: Body Mass Index; CKD-EPI: Chronic Kidney Disease Epidemiology Collaboration; HbA ${ }_{1 c}$ : Glycated Hemoglobin. $p<0.05$ is considered as statistically significant (bold).

the geographical location, Turkish people have been influenced by the European civilization and near-East Asia in terms of social, economic, genetic, and socio-cultural views. From this point of view, it can be considered as a summary of a multi-centered study.

There are studies available demonstrating that IDegAsp treatment is safer compared to basal bolus and pre-mixed insulin regimes from the point of view of hypoglycemia [7, 10, 11]. In a phase 3 study in insulin-naïve Japanese adults, IDegAsp therapy was demonstrated to have at least as hypoglycemic safety as insulin glargine U100 [12]. In another phase 3 study, it was revealed that the nocturnal confirmed hypoglycemia rate was lower with IDegAsp than biphasic insulin aspart 30 (BIAsp30) [13]. In a randomized controlled trial, IDegAsp effectively provided long-term glycemic control, and less overall and nocturnal hypoglycemia compared with BIAsp30 in patients with type 2 diabetes who were insulin-naïve [10]. In an observational study with 22 subjects, IDegAsp effectively improved glycemic index and quality of life in patients with diabetes previously treated with pre-mixed insulin [5]. In our observational study conducted with 36 participants, we included the entire patients on insulin treatment. At the beginning of the study, 29 of the patients were using metformin and it was continued. While 15 participants were using DPP4-i treatment pre-IDegAsp, it was also started for 11 ones. Whereas no participants were using SGLT2-i treatment pre-IDegAsp, it was started for 7 ones. In our study, successful results have been achieved on both hypoglycemia rate and $\mathrm{HbA}_{1 \mathrm{c}}$ levels thanks to IDegAsp supported DPP4-i or SGLT-i.

Hypoglycemia is a complication of diabetes mellitus, commonly recognized and feared, and prevents the intensification of treatment for certain patients and therefore, has a detrimental effect on quality of life and/or serious clinical outcomes [14]. Moreover, the unawareness of hypoglycemia, which is caused by the frequent severe hypoglycemia, can lead to permanent neurological damage [15]. In addition, fear of hypoglycemia leads to anxiety, behavior disorder, discontinuation of medications, loss of labor and economic decline in patients with diabetes $[16,17]$. It is acknowledged that the fear of hypoglycemia, often leads to impaired diet compliance, causes difficulty in achieving the $\mathrm{HbA}_{1 \mathrm{c}}$ target and avoids injection applications [3]. Fear of hypoglycemia, quite common in those administering oral insulin secretagogues and subcutaneous insulin therapy, has been shown to be related to age $[18,19]$. Studies also reveal that the higher the daily injection frequency and insulin doses, the greater the fear of hypoglycaemia is $[3,20]$. To the best of our knowledge, this is the first study in the literature (pubmed and google academic) on overcoming fear of hypoglycemia with IDegAsp. According to the HFS scales applied to patients at the end of our study, it was also shown that the fear of hypoglycemia was reduced in patients via IDegAsp treatment. In our study, it was also found that $\triangle$ HFS-B was associated with distinctness of daily injection frequency. There was a strong positive correlation between them (Rho: 0.398; P: 0.016). Details on the correlation of $\triangle \mathrm{HFS}$ in our study, in which no relation was shown between other parameters, is presented in Table 3.

IDegAsp serves as a simple and adaptive treatment option requiring only a single injection that can reveal a better alternative to achieve a higher standards of patients' lifestyle and QoL in comparison with more complex alternatives, with a lower frequency of daily injections 
(in comparison with the basal-bolus and premixed regimens), which is more likely to be perceived and recognized as less burdensome by patients [7, 21, 22]. By means of switching to IDegAsp in our study, the median of daily insulin administration frequency applied by the participants decreased from 4 to 2 . The entire eight patients with type 2 diabetes administered 3 times a day. As for the patients with type 2, insulin as part was administered before lunch while IDegAsp was administered before breakfast and dinner. It is likely that oral antidiabetics added to the treatment of patients with type 2 diabetes may also contribute to these outcomes. It is already apparent that there is an increase in the percentage of concomitant oral antihyperglycemic therapy after switching. Our study also reveals our clinical experience with the positioning of IDegAsp therapy in type 2 diabetes mellitus. Moreover, the IDegAsp insulin-containing pen is easier to use than other insulin pens. Accordingly, it provides ease of use for patients.

It is highly likely that the entire insulin regimens shall result in weight gain. Whereas basal insulins gain the least weight, basal-bolus treatment causes the highest weight gain [3]. In a randomized controlled study conducted with the Chinese, IDegAsp was compared to BIAsp30. Superiority for change in weight and BMI was not confirmed according to phase III, open-label, treat-to-target trial of Yang et al. [11]. In another treat-to-target trial conducted by Taneda et al., it has been observed that weight and BMI changes were similar after patients administering IDegAsp and administering BIasp30 for 26 weeks [13]. In both studies conducted by Yang and Taneda, patients receiving IDegAsp therapy were calculated for less dose of insulin administration. As compatible with the literature, in our observational study, it has been demonstrated that insulin doses were decreased after 12 months. In addition, it was measured that there was a weight loss and a decrease in BMI value at the end of the study. In these results, the effect of using SGLT2-i should not be neglected.

The first way to improve renal and cardiovascular protection is to prevent the hyperglycemia [3]. Then, it is recommended to add anti-diabetic agents (such as SGLT-i) with renoprotective effects to the treatment [3]. An improvement was observed in the CKD-EPI values of our patients who were followed up within the directives of the guidelines, even if no statistical significance was found (P: 0.122).

There are certain limitations in our study. Firstly, amount of oral antidiabetics in pre-switch and post-switch data was not similar. Since the outcomes of observational data were used, their mapping could not be appropriate. This difference actually made an additional contribution to our study for the "combined administration" of IDegAp and DPP4-i and/or SGLT2-i. Secondly, some of the laboratory parameters such as CKD-EPI are considered to be less in number of patients in order to reveal their significance more accurately. Thirdly, our study does not include a control group because of the results of observational data of patients administering IDegAsp. Fourthly, a device that continuously measures blood glucose was not employed, and the rate of hypoglycemia was assessed according to the anamnesis of the patients. So, events caused by hypoglycemia unawareness may have been off the record. Our work can be supported by further studies incorporating sensor technology in large patient groups.

In conclusion, IDegAsp co-formulation, combined with DPP4-i and/or SGLT2-i, can provide usefulness in terms of rates of hypoglycemia, reduced $\mathrm{HbA}_{1 \mathrm{c}}$, less injection administration, and decreased fear of hypoglycemia in patients with type 2 diabetes.

Research funding: None declared.

Author contributions: All authors have accepted responsibility for the entire content of this manuscript and approved its submission.

Competing interests: Authors state no conflict of interest. Informed consent: Informed consent was obtained from all individuals included in this study.

Ethical approval: The clinical trial protocol was approved by the Ethics Committee of Erciyes University and complied with the Declaration of Helsinki and Good Clinical Practice.

\section{References}

1. Kitamura T. The role of FOXO1 in $\beta$-cell failure and type 2 diabetes mellitus. Nat Rev Endocrinol 2013;9:615-23.

2. Alalwan TA, Perna S, Mandeel QA, Abdulhadi A, Alsayyad AS, D'Antona $\mathrm{G}$, et al. Effects of daily low-dose date consumption on glycemic control, lipid profile, and quality of life in adults with preand type 2 diabetes: a randomized controlled trial. Nutrients 2020; 12:217.

3. American Diabetes Association. Standards of medical care in diabetes-2017. Diabetes Care 2017;40:S11-24.

4. Sheu WH, Ji L, Lee WJ, Jabbar A, Han JH, Lew T. Efficacy and safety of premixed insulin analogs in Asian patients with type 2 diabetes: a systematic review. J Diabetes Investig 2017;8:518-34.

5. Fujimoto K, Iwakura T, Aburaya M, Matsuoka N. Twice-daily insulin degludec/insulin aspart effectively improved morning and evening glucose levels and quality of life in patients previously treated with premixed insulin: an observational study. Diabetol Metab Syndr 2018;10:64.

6. Seufert J, Borck A, Bramlage P. Addition of a single short-acting insulin bolus to basal insulin-supported oral therapy: a systematic review of data on the basal-plus regimen. BMJ Open Diabetes Res Care 2019;7:e000679. 
7. Glastras SJ, Cohen N, Dover T, Kilov G, Maclsaac RJ, McGill M, et al. The clinical role of insulin degludec/insulin aspart in type 2 diabetes: an empirical perspective from experience in Australia. J Clin Med 2020;9:1091.

8. Cox DJ, Irvine A, Gonder-Frederick L, Nowacek G, Butterfield J. Fear of hypoglycemia: quantification, validation, and utilization. Diabetes Care 1987;10:617-21.

9. Erol 0, Enc N. Hypoglycemia fear and self-efficacy of Turkish patients receiving insulin therapy. Asian Nurs Res 2011;5:222-8.

10. Franek E, Haluzík M, Canecki Varžić S, Sargin M, Macura S, Zacho J. Twice-daily insulin degludec/insulin aspart provides superior fasting plasma glucose control and a reduced rate of hypoglycaemia compared with biphasic insulin aspart 30 in insulin-naïve adults with Type 2 diabetes. Diabet Med 2016;33:497-505.

11. Yang W, Ma J, Hong T, Liu M, Miao H, Peng Y, et al. Efficacy and safety of insulin degludec/insulin aspart versus biphasic insulin aspart 30 in Chinese adults with type 2 diabetes: a phase III, open-label, 2:1 randomized, treat-to-target trial. Diabetes Obes Metab 2019;21:1652-60.

12. Onishi Y, Ono Y, Rabøl R, Endahl L, Nakamura S. Superior glycaemic control with once-daily insulin degludec/insulin aspart versus insulin glargine in Japanese adults with type 2 diabetes inadequately controlled with oral drugs: a randomized, controlled phase 3 trial. Diabetes Obes Metab 2013;15:826-632.

13. Taneda S, Hyllested-Winge J, Gall MA, Kaneko S, Hirao K. Insulin degludec/insulin aspart versus biphasic insulin aspart 30 twice daily in insulin-experienced Japanese subjects with uncontrolled type 2 diabetes: subgroup analysis of a Pan-Asian, treat-to-target Phase 3 Trial. J Diabetes 2017;9:243-7.

14. Edelman SV, Blose JS. The impact of nocturnal hypoglycemia on clinical and cost-related issues in patients with type 1 and type 2 diabetes. Diabetes Educ 2014;40:269-79.

15. Takahashi H, Nishimura R, Onda Y, Ando K, Tsujino D, Utsunomiya K. Prediction of nocturnal hypoglycemia unawareness by fasting glucose levels or post-breakfast glucose fluctuations in patients with type 1 diabetes receiving insulin degludec: a pilot study. PloS One 2017;12:e0177283.

16. Indelicato L, Mariano V, Galasso S, Boscari F, Cipponeri E, Negri C, et al. Influence of health locus of control and fear of hypoglycaemia on glycaemic control and treatment satisfaction in people with Type 1 diabetes on insulin pump therapy. Diabet Med 2017;34: 691-7.

17. Grammes J, Stock W, Mann CG, Flynn EM, Kubiak T. Focus group study to identify the central facets of fear of hypoglycaemia in people with Type 2 diabetes mellitus. Diabet Med 2017;34:1765-72.

18. Wang JS, Chen H, Tang F, Huey-Herng Sheu W. Associations of fear of hypoglycemia with second-line use of insulin secretagogues or insulin and subsequent glycemic control in patients with type 2 diabetes: an analysis using data from the DISCOVER study. Int J Clin Pract 2020:e13485.

19. Sakane N, Kotani K, Tsuzaki K, Nishi M, Takahashi K, Murata T, et al. Fear of hypoglycemia and its determinants in insulin-treated patients with type 2 diabetes mellitus. J Diabetes Investig 2015;6: 567-70.

20. Schwartz FL, Guo A, Marling CR, Shubrook JH. Analysis of use of an automated bolus calculator reduces fear of hypoglycemia and improves confidence in dosage accuracy in type 1 diabetes mellitus patients treated with multiple daily insulin injections. J Diabetes Sci Technol 2012;6:150-2.

21. Shimoda S, Sakamoto W, Hokamura A, Matsuo Y, Sekigami T, Ichimori $S$, et al. Comparison of the efficacy and safety of oncedaily insulin degludec/insulin aspart (IDegAsp) and long-acting second-generation basal insulin (insulin degludec and insulin glargine 300 units $/ \mathrm{mL}$ ) in insulin-naïve Japanese adults with type 2 diabetes: a pilot, randomized, controlled study. Endocr J 2019; 66:745-52.

22. Jang HN, Yang YS, Lee SO, Oh TJ, Koo BK, Jung HS. Favorable glycemic control with once-daily insulin degludec/insulin aspart after changing from basal insulin in adults with type 2 diabetes. Endocrinol Metab (Seoul) 2019;34:382-9. 